\title{
Using Data to "Git Gud": A Push for a Player-Centric approach to the Use of Data in Esports
}

\author{
Erica Kleinman \\ emkleinm@ucsc.edu \\ University of California, Santa Cruz \\ Santa Cruz, California
}

\author{
Magy Seif El-Nasr \\ mseifeln@ucsc.edu \\ University of California, Santa Cruz \\ Santa Cruz, California
}

\begin{abstract}
The rapid increase in the availability of player data and the advancement of player modeling technologies have resulted in an abundance of data-driven systems for the domain of esports, both within academia and the industry. However, there is a notable lack of research exploring how players use their data to gain expertise in the context of esports. In this position paper we discuss the current state of the field and argue that there is a need for further research into how players use their data and what they want from datadriven systems. We argue that such knowledge would be invaluable to better design data-driven systems that can aid players in gaining expertise and mastering gameplay.
\end{abstract}

\section{CCS CONCEPTS}

- Human-centered computing $\rightarrow$ Empirical studies in $\mathrm{HCI}$.

\section{KEYWORDS}

esports, expertise, learning, data, visualization, user studies

\section{ACM Reference Format:}

Erica Kleinman and Magy Seif El-Nasr. 2021. Using Data to "Git Gud": A Push for a Player-Centric approach to the Use of Data in Esports. In CHI 2021: ACM CHI Conference on Human Factors in Computing Systems, May 08-13, 2021, Yokohama, Japan. ACM, New York, NY, USA, 4 pages. https://doi.org/10.1145/nnnnnnn.nnnnnnn

\section{INTRODUCTION}

Data is becoming increasingly relevant in the domain of esports. On the academic side, the past decade has seen rapid advancements in player modeling techniques, visualization, and data-driven systems, spurred on by the increased availability of player data [13, 16, 24, 31]. In the industry, data mining and statistics are becoming the backbone of design and business decisions intended to keep games fun and relevant. Even for players, data has become accessible through free-to-use, player-facing data trackers and gameplay assistants $[8,26]$. The result is an environment in which players can easily reference their own data in order to track their performance over time and in relation to others, which allows them to gain expertise

Permission to make digital or hard copies of all or part of this work for personal or classroom use is granted without fee provided that copies are not made or distributed for profit or commercial advantage and that copies bear this notice and the full citation on the first page. Copyrights for components of this work owned by others than ACM must be honored. Abstracting with credit is permitted. To copy otherwise, or republish, to post on servers or to redistribute to lists, requires prior specific permission and/or a fee. Request permissions from permissions@acm.org.

CHI 2021, May 08-13, 2021, Yokohama, Japan

(c) 2021 Association for Computing Machinery.

ACM ISBN 978-x-xxxx-xxxx-x/YY/MM...\$15.00

https://doi.org/10.1145/nnnnnnn.nnnnnnn by gaining a better understanding of how a game is to be played $[7,14]$.

There is, however, something missing from this environment. While systems and methodologies for tracking and analyzing player data have grown and advanced, there is a notable lack of understanding of how players use their data to gain expertise in esports. This is because many of the tools that have been developed have not involved player input or evaluation [17, 22] or are not intended for player use at all, instead targeting developers [35] or spectators [20]. That being said, there does exist a collection of work targeting players, some of which conducted evaluations with those players, however, those evaluations focused predominantly on identifying best practices for design, comprehension, and usability [33, 37]. While this work successfully identified a number tool characteristics that support player use, such as icon design [37] or the integration of physical models [33], they do not examine the intricacies of how players use or are otherwise impacted by the data or tools.

Even in those cases where such an examination is included in the study, the discussion is high level, looking at how players used different visualizations to gain different pieces of information, and does not discuss the granularity of the interaction, or the role of data within the gameplay experience as a whole [21]. As a result, there is a notable lack of empirical study regarding the role of data and data-driven systems in esports training and gameplay, with the most relevant work on how data is used by players being predominantly theoretical $[3,23]$.

One may wonder if there are transferable findings from beyond the domain of games. A good candidate would be the related fields of EXplainable AI (XAI) and Interactive Machine Learning (IML), which have conducted extensive studies into how users use and collaborate with data-driven systems to complete various tasks $[2,41]$. However, the findings of these works are difficult to translate to the domain of games due to the inherent differences between the tasks used in the studies and games. More specifically, XAI and IML studies often use simple tasks, such as document classification [34] or email sorting [32].

In addition to being simple tasks, these are also low stakes contexts, in which a mistaken suggestion from the AI will not have a costly impact on the user. By contrast, esports are high stakes, dynamic decision making domains. Players need to think fast, account for a great deal of external factors, such as how many teammates or enemies are nearby [18], and make decisions that could determine whether they win or lose the game. Thus, we argue that, while a valuable resource, the findings of XAI and IML studies set in much simpler contexts can not be directly transferred to esports. While they can be leveraged as references to frame the study of data usage in esports expertise, we argue that the field requires its own 
exploration of how players use their data to train, learn, and inform decision making.

In this position paper, we argue that there needs to be more research that looks at gameplay data from the perspective of esports players. Specifically, we argue for player-centric research that explores, in detail (a) what players want or need from their data, (b) how players use their data, and (c) how players are impacted by their data. Further, we argue for player-centric research that explores how data-driven tools, such as visualizations or bots, can facilitate these needs and practices. We argue that data plays a key role in how players gain expertise and master gameplay in esports games, as evidenced by the abundance of player-facing data tracking systems that already exist. However, if we are to build better systems and tools that can support esport players in the use of their data, we must first turn our attention to what the players want from and do with their data.

\section{AN ABUNDANCE OF DATA}

There is an abundance of player-facing data tracking apps, such as DOTABUFF [8], OP.GG [26], and Overwatch Tracker [27], and these apps are used regularly by players to track their performance in game (personal correspondence with esports players and industry professionals, 2020). These apps specialize in presenting aggregated data, statistics, and leader-boards, and track how these change over time for individual players, making it easy for them to spot their weaknesses and make targeted decisions for how to improve play. However, these apps have also benefited research by acting as repositories from which data can be easily extracted.

Encouraged by the abundance of available data, academia and industry researchers have both produced a great variety of tools and techniques for leveraging this data towards the advancement of knowledge. Among the most popular approaches for analysis are those that leverage quantitative machine learning and statistical analysis methods to profile players [9, 29], predict victory [30, 40], and provide recommendations [4,5]. An illustrative example is the work of Drachen et al. [10] who clustered players based on spatio-temporal data from DotA2 to study patterns in how player positioning and movement correlated with the overall skill level of a team. Another example is the work of Eaton et al., who used statistical analysis to uncover correlations between victory chances and the presence and performance of important team members $[11,12]$.

Other work has taken a mixed-methods approach to analysis and modeling, combining data visualization with human insight to create human-in-the-loop systems that can help people understand gameplay $[1,18,25]$. These mixed-methods techniques are able to overcome many of the shortcomings of pure quantitative techniques, specifically, they better account for context and allow domain experts to glean insight from game data in an interactive and transparent manner [31]. Arguably, this would make such systems good candidates to aid players in their study of gameplay data in order to obtain mastery. However, these systems have been primarily developed for use in research labs, and therefore have yet to be tested with players.

\section{DATA-DRIVEN SYSTEMS FOR PLAY}

There are numerous systems and methods that have been developed with player use in mind. One key example is SENSEI [17], described as an intelligent advisory system intended to help esports players improve their gameplay through advanced analytics and ML. There is also the work of Christiansen et al. [6] who developed a novel approach for measuring the causal effects of game features or player performance on chances of winning, motivated by a desire to help players and developers better understand the connection between a given set of statistics and victory. Additionally, Chen et al. $[4,5]$ developed recommendation systems that give players recommendations on how to build better decks in card games or pick better characters in MOBAs. All of these examples involve systems designed, specifically, to help players improve at play, either by tracking their performance, helping the understand the outcomes of their choices, or recommending the best approach.

However, none of these works evaluate their systems with players, and thus, it is difficult to say the extent to which they meet players' needs. Additionally, although they are not focused directly at players, there are numerous data dashboards that have been developed for esports spectatorship, motivated by the role that spectatorship can play in mastering an esport game [19]. One key example is Weavr [20], an app that provides audiences with real time analytics, updates, and win prediction during esports tournaments. While Weavr has been evaluated in terms of how it was used and impacted the spectator experience, this focuses on spectators, rather than the players themselves, and is concerned more with how the app integrated into the experience of attending a tournament [20].

That is not to say that there is no work that empirically examines the usage of data with users specifically within the domain of games. Many examples exist, such as the work of Van den Broek et al. [33], who explored the value of including tangible elements in a data visualization system for esports, and found that most potential users saw value in the approach. Prominent in this area is the work of Wallner et al. [35-39], Kriglstein et al. [21] and Halabi et al. [15], who developed a number of different visualizations for play-testing data $[15,35,39]$ and online multiplayer game data $[21,37,38]$. The various systems were evaluated by the target users, either developers or players.

The results of these studies are valuable insights into how various visual design characteristics, such as color, opacity, shading, or icon usage, impact the clarity, informativeness, and usefulness of data visualizations for games $[15,35,37]$. However, these results are primarily contributions to the understanding and development of design best practices, and do not discuss the role of data in the player experience, or how it is used or understood by players. Even in cases that included a discussion of how users used the tools [21, 39], the observations were high level and specific to the visualization at hand. Thus, while this work presents a fantastic foundation to build upon for system design, more work is needed to understand the intricacies of how data is understood and used by players.

The key to designing better data systems for players is understanding their needs, and the key to understanding their needs when it comes to their data is understanding how they interact with, use, and make meaning from their data at a granular level. 
The work of Penney et al. [28] attempted to address this issue. They studied what information StarCraft players focused on or sought out as they interacted with a game replay, the paths they followed to acquire that information, and what decision points during play they considered to be most critical [28].

The goal of Penney et al's work was to provide a comprehensive understanding of real time strategy players' needs in regards to understanding the actions of an AI, in order to design explainable AI that better met these needs, and found that they were more interested in "what" information rather than "why" information. To our knowledge, this is the only work that examines how players explore and make sense of data in such a granular manner. However, the "data" used in the study is a match replay, closer to a video than raw data, and the goal of the study was to understand the players' needs in terms of understanding an AI's behavior, not in terms of how esports players use their data to gain expertise or the role this plays in the overall player experience.

Ultimately, there is lack of discussion of or empirical work on the role that data or a data-driven system can or does play in the overall player experience. Instead, much of the existing research that explores the role of data in the player experience does so from a theoretical manner. One such example is the work of Bowman et al. [3], who proposed a theoretical framework for visualization in games that categorized the different visualizations that exist for gameplay based on their primary purpose, target audience, temporal usage, visual complexity, and immersion/integration, which refers to whether it exists inside the game or not. However, the framework primarily focused on categorizing the types of visualizations that existed based on their purpose, and only discussed use by and impact on players at a very high level.

The work of Medler [23] presented a different theoretical framework that focused more on the player aspect, as it discussed the functionality of player dossiers, a type of data tracker that created an account of a player's experience, giving them an opportunity to learn from their past gameplay, through data, and motivating them to keep playing. However, like the work of Bowman et al., the work is purely theoretical, and no study of actual players was conducted.

\section{WHAT PLAYERS WANT FROM THEIR DATA: RESULTS FROM A PILOT STUDY}

We argue that there remains an opening and a need for research that explores, specifically, how players use their data to gain expertise in esports. Our own work exploring this topic with esports players and industry professionals has suggested that players' desires and needs, regarding their data, are nuanced. In a pilot study examining what esports players want from an $\mathrm{AI}$ assistant meant to help them play, two players who came from different gameplay backgrounds described dramatically different tools (personal communications, 2020).

The first player, with experience playing multiplayer online battle arena (MOBA) games, described a tool that could give quick advice during play, coupled with detailed reports post game. He specifically focused on a need for decision support backed up by descriptive stats, stating "Statistics will support the bots suggestions so if you have numbers then the players would be more ready to accept that particular suggestion." Similarly, he discussed an inability to trust a tool that did not understand the game as well as he did, stating "if you are at rank 5 and, rank one being the best rank the topmost rank. I remember [this bot] telling me that even though I am rank five my game play was like rank six level and I was like please no don't tell me how to play this game and I just deleted that bot".

The second player, with experience in online competitive card games, described a tool that could learn to play like a human and be trained against. He specifically emphasized improving his gameplay play and practice with a human-like opponent, stating "let's say this AI I turn it on and I play 20 games against you know this one friend of mine TJ...it just kind of sees what TJ regularly does and then I have this bot that kind of simulates a lot of their reactions." Of note, however, and contrary to the desires of the first player, was that this player also expressed an aversion to any tool that felt like it was making decisions in his place, stating "an algorithm is making a choice for me and that again is similar to the idea of meta decking that is me using someone else's abilities you know in building a deck to win which to me doesn't feel right."

The results of this pilot highlight two key points. First, players are open to using data and data-driven systems to train and master gameplay. In fact, as both pilot participants revealed, many of them already do. This finding is supported by the many companies who have invested in and made a business out of the development of state-of-the-art data tracking and modeling tools that professional esports teams can use for training (personal communication with industry professional). The second key point, however, is that players' desires and needs are nuanced and dependent on individual differences. This comes as no surprise, as individual differences always impact play [31]. However, these nuances need to be better understood if we intend to design systems to help players gain expertise, and in order to better understand these nuances, we need to better understand how players are using their data.

\section{CONCLUSION}

We believe that there needs to be more research that focuses specifically on how players use their data to gain expertise in esports games. In this position paper, we present an overview of the current state of work exploring data in games and esports. An abundance of data has led to a rapid expansion in novel techniques for analysis and modeling and unique player-facing systems meant to aid and train. Further, a great deal of research explores data-driven systems for games in terms of what design properties help with clarity and usability. Related fields, such as XAI and IML, also provide a rich body of knowledge that can serve as a foundation for understanding human interaction with data-driven systems.

That being said, significantly less research takes an empirical and granular look at the role of such systems, and the data they depict, within the esports player experience. More specifically, there is little to know work examining how players use their data to gain expertise, and the impact this has on their experience. In order to better inform the design of data-driven systems for esports, we propose that we need a more thorough understanding of the role of data in the esports in terms of what players want from their data, how they use it to gain expertise, and how it impacts their experience. 


\section{ACKNOWLEDGMENTS}

This work is partially supported by the National Science Foundation under Grant Number 1917855. The authors would like to thank all current members of the project.

\section{REFERENCES}

[1] Sabbir Ahmad, Andy Bryant, Erica Kleinman, Zhaoqing Teng, Truong-Huy D Nguyen, and Magy Seif El-Nasr. 2019. Modeling Individual and Team Behavior through Spatio-temporal Analysis. In Proceedings of the Annual Symposium on Computer-Human Interaction in Play. ACM, 601-612.

[2] Saleema Amershi, Maya Cakmak, William Bradley Knox, and Todd Kulesza. 2014 Power to the people: The role of humans in interactive machine learning. $A i$ Magazine 35, 4 (2014), 105-120.

[3] Brian Bowman, Niklas Elmqvist, and TJ Jankun-Kelly. 2012. Toward visualization for games: Theory, design space, and patterns. IEEE transactions on visualization and computer graphics 18, 11 (2012), 1956-1968.

[4] Zhengxing Chen, Christopher Amato, Truong-Huy D Nguyen, Seth Cooper Yizhou Sun, and Magy Seif El-Nasr. 2018. Q-deckrec: A fast deck recommendation system for collectible card games. In 2018 IEEE Conference on Computational Intelligence and Games (CIG). IEEE, 1-8.

[5] Zhengxing Chen, Truong-Huy D Nguyen, Yuyu Xu, Christopher Amato, Seth Cooper, Yizhou Sun, and Magy Seif El-Nasr. 2018. The art of drafting: a teamoriented hero recommendation system for multiplayer online battle arena games. In Proceedings of the 12th ACM Conference on Recommender Systems. ACM, 200208.

[6] Anders Harboell Christiansen, Emil Gensby, and Bryan S Weber. 2019. Resolving Simultaneity Bias: Using Features to Estimate Causal Effects in Competitive Games. In 2019 IEEE Conference on Games (CoG). IEEE, 1-8.

[7] Scott Donaldson. 2017. Mechanics and metagame: Exploring binary expertise in League of Legends. Games and Culture 12, 5 (2017), 426-444.

[8] dotabuff. 2021. DOTABUFF. https://www.dotabuff.com/. Online; accessed 25 January 2021.

[9] Anders Drachen, Rafet Sifa, Christian Bauckhage, and Christian Thurau. 2012 Guns, swords and data: Clustering of player behavior in computer games in the wild. In 2012 IEEE conference on Computational Intelligence and Games (CIG). IEEE, 163-170.

[10] Anders Drachen, Matthew Yancey, John Maguire, Derrek Chu, Iris Yuhui Wang Tobias Mahlmann, Matthias Schubert, and Diego Klabajan. 2014. Skill-based differences in spatio-temporal team behaviour in defence of the ancients 2 (dota 2). In 2014 IEEE Games Media Entertainment. IEEE, 1-8.

[11] Joshua A Eaton, David J Mendonça, and Matthew-Donald D Sangster. 2018 Attack, Damage and Carry: Role Familiarity and Team Performance in League of Legends. In Proceedings of the Human Factors and Ergonomics Society Annual Meeting, Vol. 62. SAGE Publications Sage CA: Los Angeles, CA, 130-134.

[12] Joshua A Eaton, Matthew-Donald D Sangster, Molly Renaud, David J Mendonca, and Wayne D Gray. 2017. Carrying the Team: The Importance of One Player's Survival for Team Success in League of Legends. In Proceedings of the Human Factors and Ergonomics Society Annual Meeting, Vol. 61. SAGE Publications Sage CA: Los Angeles, CA, 272-276.

[13] Magy Seif El-Nasr, Anders Drachen, and Alessandro Canossa. 2013. Game analytics. Springer.

[14] Joey R Fanfarelli. 2018. Expertise in professional overwatch play. International Journal of Gaming and Computer-Mediated Simulations (IFGCMS) 10, 1 (2018), $1-22$.

[15] Nour Halabi, Günter Wallner, and Pejman Mirza-Babaei. 2019. Assessing the impact of visual design on the interpretation of aggregated playtesting data visualization. In Proceedings of the Annual Symposium on Computer-Human Interaction in Play. 639-650.

[16] Danial Hooshyar, Moslem Yousefi, and Heuiseok Lim. 2019. A systematic review of data-driven approaches in player modeling of educational games. Artificial Intelligence Review 52, 3 (2019), 1997-2017.

[17] Andrzej Janusz, Dominik Slezak, Sebastian Stawicki, and Krzysztof Stencel. 2018 SENSEI: an intelligent advisory system for the esport community and casual players. In 2018 IEEE/WIC/ACM International Conference on Web Intelligence (WI) IEEE, 754-757.

[18] Erica Kleinman, Sabbir Ahmad, Zhaoqing Teng, Andy Bryant, Truong-Huy D. Nguyen, Casper Harteveld, and Magy Seif El-Nasr. 2020. "And Then They Died": Using Action Sequences for Data Driven, Context Aware Gameplay Analysis. In International Conference on the Foundations of Digital Games (Bugibba, Malta) (FDG '20). Association for Computing Machinery, New York, NY, USA, Article 63 , 12 pages. https://doi.org/10.1145/3402942.3402962

[19] Erica Seif Kleinman and Magy El-Nasr. 2020. Interactive Visualization For Strategy Acquisition In Esports Spectatorship. In Seegames Workshop, CHI 2020.
[20] Athanasios Vasileios Kokkinakis, Simon Demediuk, Isabelle Nölle, Oluseyi Olarewaju, Sagarika Patra, Justus Robertson, Peter York, Alan Pedrassoli Pedrassoli Chitayat, Alistair Coates, Daniel Slawson, et al. 2020. DAX: Data-Driven Audience Experiences in Esports. In ACM International Conference on Interactive Media Experiences. 94-105.

[21] Simone Kriglstein, Günter Wallner, and Margit Pohl. 2014. A user study of different gameplay visualizations. In Proceedings of the SIGCHI Conference on Human Factors in Computing Systems. 361-370.

[22] Crisrael Lucero, Christianne Izumigawa, Kurt Frederiksen, Lena Nans, Rebecca Iden, and Douglas S Lange. 2020. Human-Autonomy Teaming and Explainable AI Capabilities in RTS Games. In International Conference on Human-Computer Interaction. Springer, 161-171.

[23] Ben Medler. 2011. Player dossiers: Analyzing gameplay data as a reward. Game Studies 11, 1 (2011).

[24] Benedikte Mikkelsen, Christoffer Holmgård, and Julian Togelius. 2017. Ethical considerations for player modeling. In Workshops at the Thirty-First AAAI Conference on Artificial Intelligence.

[25] Dinara Moura, Magy Seif el Nasr, and Christopher D Shaw. 2011. Visualizing and understanding players' behavior in video games: discovering patterns and supporting aggregation and comparison. In Proceedings of the 2011 ACM SIGGRAPH symposium on video games. ACM, 11-15.

[26] op.gg. 2021. op.gg. https://na.op.gg/. Online; accessed 25 January 2021.

[27] Overwatch Tracker. 2021. OVERWATCH TRACKER. https://https:// overwatchtracker.com/. Online; accessed 25 January 2021.

[28] Sean Penney, Jonathan Dodge, Claudia Hilderbrand, Andrew Anderson, Logan Simpson, and Margaret Burnett. 2018. Toward foraging for understanding of StarCraft agents: An empirical study. In 23rd International Conference on Intelligent User Interfaces. 225-237.

[29] Daniel Ramirez-Cano, Simon Colton, and Robin Baumgarten. 2010. Player classification using a meta-clustering approach. In Proceedings of the 3rd Annual International Conference Computer Games, Multimedia \& Allied Technology. 297304.

[30] Matthias Schubert, Anders Drachen, and Tobias Mahlmann. 2016. Esports analytics through encounter detection. In Proceedings of the MIT Sloan Sports Analytics Conference, Vol. 1. 2016

[31] Magy Seif El-Nasr and Erica Kleinman. 2020. Data-Driven Game Development: Ethical Considerations. In International Conference on the Foundations of Digital Games. 1-10.

[32] Alison Smith-Renner, Ron Fan, Melissa Birchfield, Tongshuang Wu, Jordan BoydGraber, Daniel S Weld, and Leah Findlater. 2020. No explainability without accountability: An empirical study of explanations and feedback in interactive $\mathrm{ml}$. In Proceedings of the 2020 CHI Conference on Human Factors in Computing Systems. 1-13.

[33] Wesley van den Broek, Günter Wallner, and Regina Bernhaupt. 2019. ModataImproving Dota 2 Experience and Spectatorship through Tangible Gameplay Visualization. In Extended Abstracts of the Annual Symposium on ComputerHuman Interaction in Play Companion Extended Abstracts. 723-730.

[34] Emily Wall, Soroush Ghorashi, and Gonzalo Ramos. 2019. Using expert patterns in assisted interactive machine learning: A study in machine teaching. In IFIP Conference on Human-Computer Interaction. Springer, 578-599.

[35] Günter Wallner, Nour Halabi, and Pejman Mirza-Babaei. 2019. Aggregated visualization of playtesting data. In Proceedings of the 2019 CHI Conference on Human Factors in Computing Systems. 1-12.

[36] Günter Wallner and Simone Kriglstein. 2012. A spatiotemporal visualization approach for the analysis of gameplay data. In Proceedings of the SIGCHI conference on human factors in computing systems. 1115-1124.

[37] Guenter Wallner and Simone Kriglstein. 2016. Visualizations for retrospective analysis of battles in team-based combat games: A user study. In Proceedings of the 2016 Annual Symposium on Computer-Human Interaction in Play. 22-32.

[38] Günter Wallner and Simone Kriglstein. 2020. Multivariate Visualization of Game Metrics: An Evaluation of Hexbin Maps. In Proceedings of the Annual Symposium on Computer-Human Interaction in Play. 572-584.

[39] Günter Wallner, Simone Kriglstein, Florian Gnadlinger, Michael Heiml, and Jochen Kranzer. 2014. Game user telemetry in practice: A case study. In Proceedings of the 11th Conference on Advances in Computer Entertainment Technology. $1-4$

[40] Pu Yang, Brent E Harrison, and David L Roberts. 2014. Identifying patterns in combat that are predictive of success in MOBA games.. In FDG.

[41] Yunfeng Zhang, Q Vera Liao, and Rachel KE Bellamy. 2020. Effect of confidence and explanation on accuracy and trust calibration in ai-assisted decision making. In Proceedings of the 2020 Conference on Fairness, Accountability, and Transparency. 295-305. 